\title{
Determinación de un modelo predictivo de secado para la manzana producida en la sierra centro.
}

Determination of a predictive drying model for the apple, produced in central highlands.

Sandra Elizabeth López Sampedro. ${ }^{1}$. Ana Cristina León Naranjo. ${ }^{2}$. Darío Javier Baño Ayala. ${ }^{3}$ \& Luis Fernando Arboleda Alvarez. ${ }^{4}$

Abstract.

Introduction. The determination of a mathematical model to predict the drying of a product is of great importance to establish industrialization processes in this case of the apple produced in the central highlands Zone 3. Objective. Establish optimal temperature and time conditions for the apple drying process. Methodology. Statistically, a completely randomized design was used with three temperatures $\left(45^{\circ} \mathrm{C}, 55^{\circ} \mathrm{C}\right.$, and 65 ${ }^{\circ} \mathrm{C}$ ) and three repetitions each. The following tests were carried out on the final product: physical-chemical: Brix, acidity, $\mathrm{pH}$, ash, humidity, dry matter as well as microbiological and sensorial. Results. The best results were $6.30^{\circ}$ Brix at $65^{\circ} \mathrm{C}$, for acidity the best results were at $55^{\circ} \mathrm{C}$ with responses equal to 0.62 . For dry matter and sensory tests the best temperature was $65^{\circ} \mathrm{C}$. The microbiological analyzes showed the absence of microorganisms; The economic analysis showed that the best treatment was at $65^{\circ} \mathrm{C}$, it was then possible to determine the drying ratio in the apple using the mathematical model based on the equation $\mathrm{Y}=\mathrm{a}+\mathrm{P} 1$ (b1) $+\mathrm{MR}$ (b2) to improve efficiency in drying.

\footnotetext{
1 Escuela Superior Politécnica de Chimborazo, Facultad de Ciencias Pecuarias. Riobamba, Ecuador.salopez@espoch.edu.ec, https://orcid.org/0000-0003-0209-2087

2 Profesional Independiente, Riobamba, Ecuador. wichyad@gmail.com

3 Escuela Superior Politécnica de Chimborazo, Facultad de Ciencias Pecuarias. Riobamba, Ecuador. dbano@yahoo.es https://orcid.org/0000-0003-0209-2087

4 Escuela Superior Politécnica de Chimborazo, Facultad de Ciencias Pecuarias. Riobamba, Ecuador. luisf.arboleda@espoch.edu.ec, https://orcid.org/0000-0001-5541-6239
} 
Conclusion. It was concluded that the temperature of $65^{\circ} \mathrm{C}$ presents better results in terms of final mass after dehydration

Keywords: Fruit, apple, drying model, temperature.

\section{Resumen}

Introducción. la determinación de un modelo matemático para predicción del secado de un producto resulta de gran importancia para establecer procesos de industrialización en este caso de la manzana producida en la sierra centro Zona 3. Objetivo. Establecer condiciones de temperatura y tiempo óptimas para el proceso de secado de la manzana. Metodología. Estadísticamente se empleó un diseño completamente al azar con tres temperaturas $\left(45^{\circ} \mathrm{C}, 55^{\circ} \mathrm{C}\right.$, y $\left.65^{\circ} \mathrm{C}\right)$ y tres repeticiones cada una. En el producto final se realizaron los siguientes ensayos: físico químicos: Brix, acidez, $\mathrm{pH}$, cenizas, humedad, materia seca además de microbiológicos y sensoriales. Resultados. Los mejores resultados fueron $6,30^{\circ}$ Brix a $65^{\circ} \mathrm{C}$, para acidez los mejores resultaros fueron a $55^{\circ} \mathrm{C}$ con respuestas iguales a 0,62. Para materia seca y pruebas sensoriales la mejor temperatura fue $65^{\circ} \mathrm{C}$. Los análisis microbiológicos mostraron ausencia de microorganismos; el análisis económico mostró que el mejor tratamiento fue a $65^{\circ} \mathrm{C}$, se pudo entones determinar el ratio de secado en la manzana utilizando el modelo matemático basado en la ecuación $\mathrm{Y}=\mathrm{a}+\mathrm{P} 1$ (b1)+MR (b2) para mejorar la eficiencia en el secado Conclusión. Se concluyó que la temperatura de $65^{\circ} \mathrm{C}$ presenta mejores resultados en cuanto a masa final posterior al deshidratado

Palabras claves: Fruta, manzana, modelo de secado, temperatura.

\section{Introducción.}

El Ecuador presenta un cultivo de manzana ancestral como su historia misma, generando diversas técnicas que mediante la producción adecuada ha ayudado a cubrir la demanda surgida a través de los años. Además, representa un ecosistema con una alta riqueza natural, por la presencia de la Cordillera de los Andes que ha influido en este factor ecosistémico (León 2019, citado en Montalván 2014).

Como segundo factor se tiene a la posición geográfica con base en el cultivo, logrando así una producción local autosuficiente localizada en la región centro del ecuador o zona 3, principalmente provincia de Tungurahua (León 2019 citado en Paredes, 2019).

La diversidad frutícola y productiva de Tungurahua se ha visto amenazada durante los últimos años debido a varios factores como la baja productividad, las importaciones de fruta, el descuido de las partes interesadas y continuas erupciones volcánicas. Siendo la variedad de manzana Emilia, una de las frutas mayormente afectadas a pesar de ser considerada un símbolo de cultura y tradición entre sus pueblos (León 2019 citado en Lara, 2015; Sánchez 2016). 
La deshidratación como tratamiento de conservación de productos agrícolas se ha empleado ampliamente en frutas, es así que con el fin de mejorar la eficiencia en el secado, se evaluaron tres temperaturas experimentales para ajustarlas a un modelo de predicción matemático

\section{Metodologia.}

La presente investigación se realizó en la Facultad de Ciencias Pecuarias de la Escuela Superior Politécnica de Chimborazo (ESPOCH). El análisis proximal y fisicoquímico se realizó en el Laboratorio de Alimentos y Conservas, Laboratorio de Microbiología y Bromatología de la Facultad de Ciencias Pecuarias, ESPOCH, ubicado en el cantón Riobamba Kilómetro 1 1/2 Panamericana sur. La investigación tendrá un tiempo de duración estimado de 90 días.

Se consideraron dos parámetros de estudio: i) temperaturas de 45,55 y $65^{\circ} \mathrm{C}$ y ii) tiempos de 3, 6 y 9 horas, con lo cual se determinó las condiciones óptimas para el secado de la cantidad de materia prima a ser utilizada. Se realizaron pruebas de laboratorio como: Análisis Sensorial: color, olor, sabor y textura; Características Fisicoquímicas: humedad, cenizas, ${ }^{\circ}$ Brix, pH); Análisis Microbiológico: mohos, levaduras. Para la evaluación de las características físicas y sensoriales de la manzana deshidratada se evaluaron diferentes temperaturas que constituyeron el factor A y diferentes tiempos como factor B. Las unidades experimentales fueron modeladas en un diseño completamente al azar en arreglo bifactorial.

Para la determinación de la significancia de las variables sensoriales se utilizó una estadística descriptiva de las degustaciones de acuerdo con criterios de evaluación entre la mayor y menor preferencia y los resultados fueron ilustrados utilizando gráficos porcentuales. Los resultados experimentales fueron modelados utilizando un diseño completamente al azar simple utilizando el programa estadístico INFOSTAT. Los análisis estadísticos fueron: Análisis de varianza (ADEVA), separación de medias, la prueba de Tukey al 0,05 de significancia.

\section{Resultados y discusión}

\section{Ratio del Secado}

Se realizó un secado de manzana de 45,55 y $65^{\circ} \mathrm{C}$ hasta conseguir pesos constantes.

Cuadro1: Ratio de secado de la manzana a $45^{\circ} \mathrm{C}$

\begin{tabular}{cc}
\hline Temperatura & $45^{\circ} \mathrm{C}$ \\
\hline Masa relativa & 0,11 gramos \\
Constante A & 0,0095 \\
Constante B & $-0,224$ \\
Tiempo & 10 horas \\
\hline
\end{tabular}


Cuadro 2: Ratio de secado de la manzana $55^{\circ} \mathrm{C}$

\begin{tabular}{cc}
\hline Temperatura & $55^{\circ} \mathrm{C}$ \\
\hline Masa relativa & 0,11 gramos \\
Constante A & $-0,1896$ \\
Constante B & $-0,2687$ \\
Tiempo & 8 horas \\
\hline
\end{tabular}

Cuadro 3: Ratio de secado de la manzana a $65^{\circ} \mathrm{C}$

\begin{tabular}{ll}
\hline Temperatura & $65^{\circ} \mathrm{C}$ \\
\hline Masa relativa & 0,11 gramos \\
Constante A & $-0,2936$ \\
Constante B & $-0,3347$ \\
Tiempo & 6 horas \\
\hline
\end{tabular}

El tiempo utilizado en el secado de manzana para $45^{\circ} \mathrm{C}$ fue de 10 horas; para $55^{\circ} \mathrm{C}, 8$ horas y para $65^{\circ}, 6$ horas. Estos valores coinciden con los obtenidos en la parte experimental a partir de estas horas establecidas por el modelo los pesos son constantes. El ratio de secado es utilizado para estandarizar el secado en material vegetal, de manera que puedan ser utilizadas a diferentes condiciones de humedad relativa, velocidad del aire y temperatura. (León 2019 citado en Hernández 2018, Jaramillo 2012)

\section{Grados Brix}

En el gráfico 1, al analizar la variable de grados Brix se observó que existen diferencias altamente significativas para la interacción $\mathrm{AxB}(\mathrm{P}<0.01)$ Al comparar los valores de los tres tratamientos $\left(45^{\circ} \mathrm{C}, 55^{\circ} \mathrm{C}, 65^{\circ} \mathrm{C}\right)$ a 9 horas de deshidratación se observó que no hay diferencias estadísticas. De acuerdo con el análisis de regresión se observó una respuesta cuadrática $(\mathrm{P}<0.04), \mathrm{y}=0,019 \mathrm{x} 2-2,130 \mathrm{x}+60,55$ con un coeficiente de determinación de $36.7 \%$.

Gráfico 1: Contenido de ${ }^{\circ}$ Brix presentes en la Manzana deshidratada.

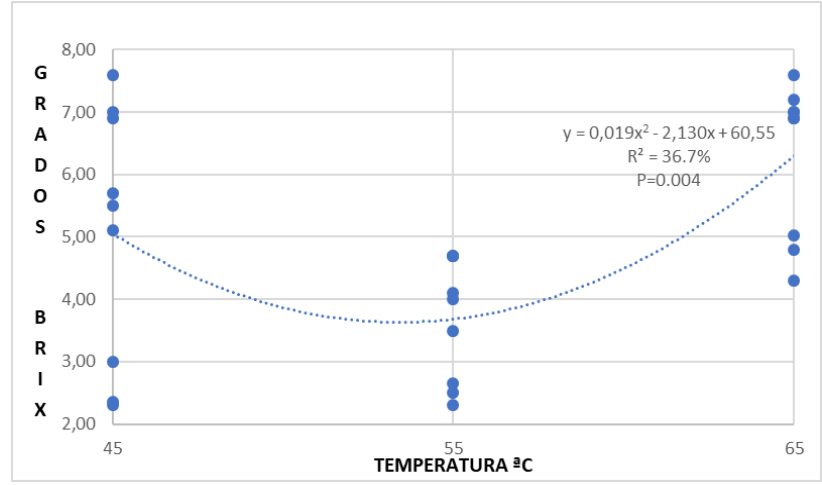


De acuerdo a la norma española de deshidratado de frutas (NTC 5468, 2007), el mínimo de ${ }^{\circ}$ Brix que debe contener una fruta deshidratada es de $\left(10^{\circ} \mathrm{Brix}\right)$. Los resultados que se obtuvieron en esta investigación son inferiores a los requerimientos.

Según lo estipulado por en un estudio proceso de deshidratación osmótica y por flujo de aire caliente de la fruta bomba (Carica papaya L.) variedad Maradol roja. Estos estipularon una temperatura de secado de $60{ }^{\circ} \mathrm{C}$ durante un tiempo de 5 horas y una concentración de sacarosa de $70{ }^{\circ}$ Brix (León 2019 citado en Contreras, 2014).

En su estudio realizado por Contreras 2014, en el cual concluye que la temperatura de secado fue el factor de mayor efecto sobre la variable de tiempo de secado de las láminas, donde el nivel alto de $60^{\circ} \mathrm{C}$ registró un tiempo de nueve horas y una menor humedad final.

\section{Acidez total}

En la gráfica 2, se observa que no existen diferencias significativas entre valores de acidez provenientes de los diferentes tratamientos térmicos.

Los valores más altos corresponden a los tratamientos: $45^{\circ} \mathrm{C}, 55^{\circ} \mathrm{C}$ y $65^{\circ} \mathrm{C}-3,6$ y 9 horas de deshidratado respectivamente de la manzana. Mientras que el menor contenido de acidez $(0,58)$ se observó cuando se utilizó una temperatura a $45^{\circ} \mathrm{C}$ y por un tiempo de 6 horas.

Gráfico 2: Acidez en la manzana deshidratada.

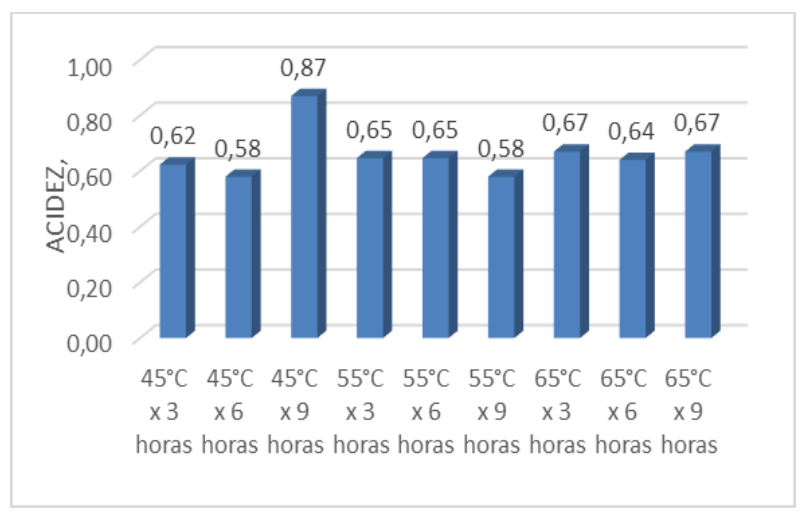

Fuente: Autor, (2019).

La variabilidad que existió en la acidez de las muestras al atravesar los distintos rangos de temperatura fue muy notoria. A pesar de que a una mayor temperatura los niveles de pH bajan, en el caso de una temperatura de $65{ }^{\circ} \mathrm{C}$ 'el nivel de acidez se elevó, puede ser debido a las reacciones de degradación térmica de los ácidos orgánicos y vitaminas presentes en la fruta entre otros factores. (León 2019 citado en Cabezas 2014; Alcántara 2007). 
De acuerdo a lo que reporta la norma (NTC 5468, 2007) indica que el valor máximo es (3.3\%) de acidez para frutas ácidas. Sin embargo, los valores son relativamente bajos. En el trabajo de investigación "Deshidratación Osmótica y Secado por Aire Caliente en Mango, Guayaba y Limón para la Obtención de Ingredientes Funcionales" en el cual se obtiene una cantidad de 0,46 y 1,17 de acidez para el mango y la guayaba respectivamente además de lo descrito por (León 2019 citado en Amador 2009).

\section{pH}

$\mathrm{Al}$ analizar los resultados obtenidos del potencial de hidrogeno $(\mathrm{pH})$ y la interacción de la temperatura/ tiempo de deshidratación, se puede observar que existen diferencias significativas de 0,08 entre 3 a 9 horas de deshidratación. En el gráfico 3-18 el menor valor de $\mathrm{pH}$ fue de 0,63 a una temperatura de $45^{\circ} \mathrm{C}$ por 3 horas de secado, y el resultado más alto a $65^{\circ} \mathrm{C}$ por 3 horas de deshidratación el $\mathrm{pH}$ fue de 4,87 . A un mayor tiempo de estadía en el deshidratador, las muestras alcanzaron un mayor $\mathrm{pH}$, teniendo diversos factores que podrían haber contribuido a estos valores principalmente la degradación térmica (León 2019 citado en Bastidas, 2014).

De acuerdo con el análisis de regresión se observó una respuesta cuadrática $(\mathrm{P}<0.03)$, y $=-0,003 \times 2+0,451 \times-10,30$ con un coeficiente de determinación $25.9 \%$.

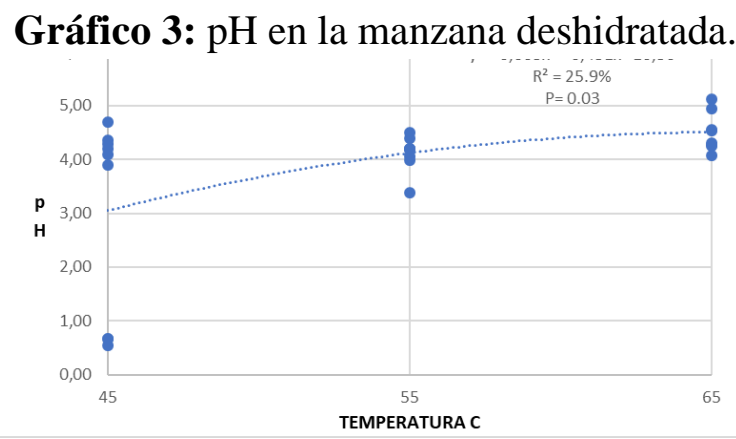

Fuente: Autor, (2019).

La variabilidad que existió en el pH de las muestras al atravesar los distintos rangos de temperatura fue muy notoria, quedando a una temperatura de $65{ }^{\circ} \mathrm{C}$ una cantidad de $\mathrm{pH}$ de 4,48; lo cual se puede corroborar en el estudio realizado por (León 2019 citado en Núñez F, 2019) "Empleo del método de secado convectivo combinado para la deshidratación de papaya (Carica papaya L.), variedad Maradol roja" en el cual utilizó una temperatura de $60{ }^{\circ} \mathrm{C}$, obteniendo una cantidad de $\mathrm{pH}$ medido de 5,65 (León 2019 citado en Cabezas, 2014).

De acuerdo a los reportes de la norma de deshidratado de frutas (NTC 5468, 2007 y Cerezo 2012) indica que el mínimo de $\mathrm{pH}$ es 3.8. Al observar los resultados obtenidos de la manzana deshidratada, en los tres tratamientos a 9 horas de deshidratado estos se encuentran dentro de la norma. 


\section{Contenido de cenizas.}

El contenido de cenizas (\%) encontrado en la manzana deshidratada presentó diferencias altamente significativas $(\mathrm{P}<0,01)$, por consecuencia de la interacción entre la temperatura y el tiempo de deshidratación, en el gráfico 4 se indica los resultados obtenidos a temperaturas $\left(45^{\circ} \mathrm{C}, 55^{\circ} \mathrm{C}\right.$ y $\left.65^{\circ} \mathrm{C}\right)$.

Con un contenido de ceniza de $1.49 \%, 3.26 \%$ y $4.36 \%$ a un tiempo de 9,6 y 6 horas de secado respectivamente, y el menor porcentaje de ceniza $(0,30 \%)$ a $45^{\circ} \mathrm{C}$ a un tiempo de 3 horas de desecado a una temperatura de $65^{\circ} \mathrm{C}$ y un tiempo de 6 horas, las muestras de manzana arrojaron el valor más alto de cenizas con un 4,36 \%.

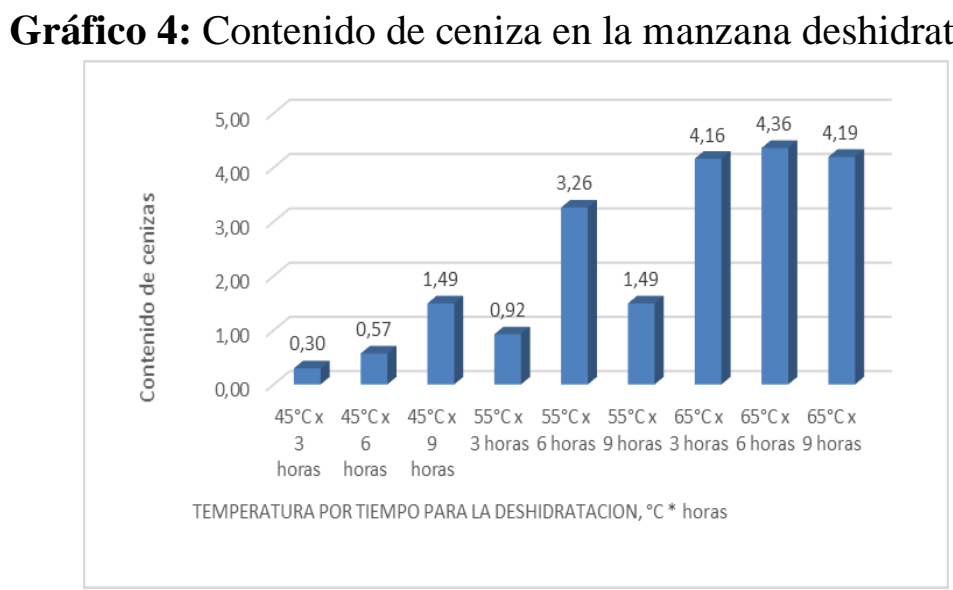

Fuente: Autor, (2019).

A un mayor tiempo de estadía en el deshidratador, las muestras alcanzaron un mayor contenido de ceniza, teniendo diversos factores que podrían haber contribuido a estos valores.

Los resultados se pueden corroborar en el estudio realizado por (León 2019 citado en Contreras, 2014) "Evaluación de métodos de deshidratación en pitahaya, para el aprovechamiento de fruta que no reúne estándares de exportación en fresca" en el cual se obtuvo para los dos métodos de secado un porcentaje de ceniza de 3,89 y 2,1\%.

\section{Contenido de Humedad.}

El porcentaje de humedad se puede observar en el gráfico 5, donde se muestran los resultados obtenidos, considerando los mejores resultados en los tres tratamientos $\left(45^{\circ} \mathrm{C}\right.$, $55^{\circ} \mathrm{C}$ y $65^{\circ} \mathrm{C}$ ) a 6 y 9 horas de secado, con un promedio de humedad de $19.89 \%, 21.85 \%$, $22,40 \%$ respectivamente, se puede observar el valor más alto $(22,40)$ cuando se utilizó una temperatura de $65^{\circ} \mathrm{C}$ por un tiempo de 9 horas, respuestas que pueden demostrar que a un mayor tiempo de estadía en el deshidratador, las muestras alcanzaron un mayor contenido de humedad (León 2019 citado en Fito, 2019) 
Gráfico 5. Contenido de humedad interacción de temperaturas y tiempos de deshidratación.

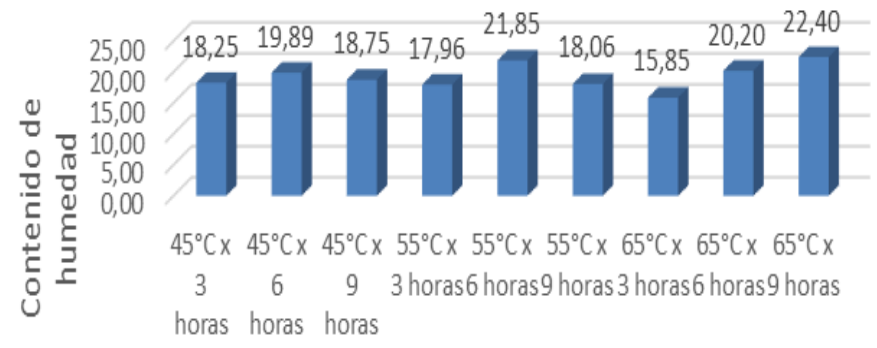

Fuente: Autor, (2019).

En el análisis de varianza de los resultados existen diferencia altamente significativas $(\mathrm{P}<0,01)$ entre la interacción de temperatura y el tiempo de secado. A medida que el tiempo y la temperatura de deshidratado se incrementa, el porcentaje de humedad disminuye. Los resultados obtenidos en esta investigación son mayores en comparación a los que reporta la norma (NTC 5468, 2007), la cual indica que el porcentaje máximo de humedad debe ser de $15 \%$, estos valores obtenidos en el estudio son adecuados para asegurar la calidad microbiológica del producto deshidratado (León 2019 citado en Gómez, 2015).

\section{Contenido de Materia Seca.}

El contenido de materia seca de la manzana deshidratada por consecuencia de la interacción entre la temperatura y el tiempo de deshidratación, presentaron diferencias altamente significativas $(\mathrm{P}<0,01)$, obteniendo los mejores resultados a temperaturas de $55^{\circ} \mathrm{C}, 65^{\circ} \mathrm{C}$ a 3 horas de deshidratado con valores de $82.04 \%$ y $84.15 \%$ de materia seca. Mientras que el menor contenido de materia seca fue de $77,60 \%$ a una temperatura de $65^{\circ} \mathrm{C}$ y por un tiempo de 9 horas, como se observa en el gráfico. (León, 2019)

Gráfico 6. Materia seca de la manzana (Malus Communis. Sp) interacción de temperaturas y tiempos de secado.

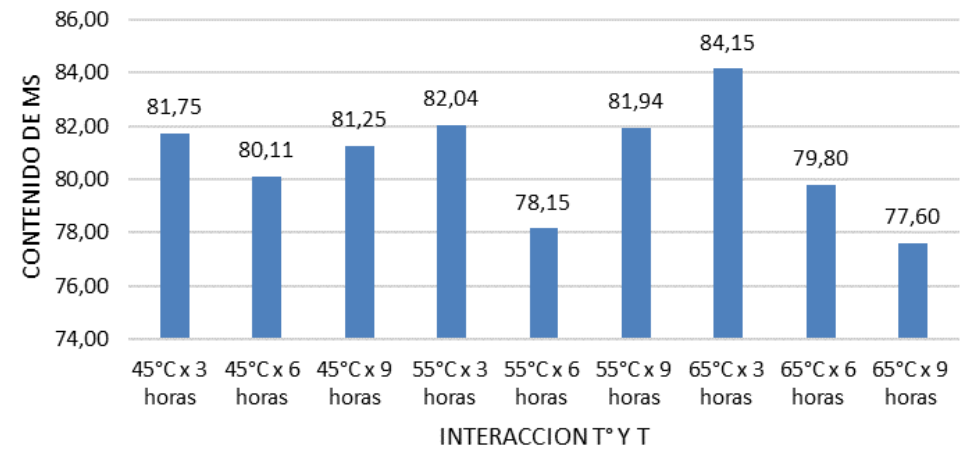

El contenido de materia seca se incrementa por la evaporación del agua presente en la fruta, en esta investigación el contenido de materia seca es menor a la descrita lo que reporta el agua es el componente más abundante de los frutos, encontrándose en niveles 
comprendidos entre 89 y $94 \%$ de materia seca, estos valores pueden depender al estado de madurez de la fruta (León 2019 citado en Cabezas, 2014; Hernández 2014).

La variabilidad que existió en la materia seca de las muestras al atravesar los distintos rangos de temperatura fue bajamente notorio, quedando a una temperatura de $65{ }^{\circ} \mathrm{C}$ una cantidad aceptable de materia seca, lo cual se puede corroborar en el estudio realizado por (León 2019 citado en Núñez F, 2019) "Empleo del método de secado convectivo combinado para la deshidratación de papaya (Carica papaya L.), variedad Maradol roja" en el cual utilizó una temperatura de $60^{\circ} \mathrm{C}$, obteniendo una cantidad de materia seca de $90,8 \%$.

\section{Mohos y Levaduras.}

Para evaluar la calidad microbiológica para la muestra de manzana deshidratada fue necesario realizar un análisis microbiológico para determinar la presencia de microorganismos que podrían afectar no solo en la calidad del producto, sino también para su uso como alimento. De esta manera, se obtuvieron valores satisfactorios, siendo que las muestras fueron sometidas a temperaturas de 45,55 y $65^{\circ} \mathrm{C}$ a tiempos de 3,6 y 9 horas. Cada muestra dio resultado negativo a la presencia de mohos y levaduras, indicando una concentración de $0 \mathrm{UP} / \mathrm{cm}^{3}$ cumpliendo con los requisitos establecidos por la norma, la cual indica que una muestra alimenticia podría presentar un valor $<10$ para mohos y levaduras. (León, 2019)

\section{Análisis Microbiológico.}

\section{Color}

En el grafico 7, se observa que en el tratamiento con temperatura de secado $55^{\circ} \mathrm{C}$ es el tratamiento que más aceptabilidad en color tuvo por los catadores. El 40,9\% de ellos afirmó que el color de la fruta les gusta; el 27,3\% no les gustó ni les disgustó; el $31.8 \%$ no les gustó. Seguido del tratamiento con temperatura de secado $65^{\circ} \mathrm{C}$, el $40,9 \%$ señalo que el color de la fruta deshidratada les gustó; el 36,4 \% no les gustó ni disgustó, y el 22,7\% no les gustó. (León, 2019)

Gráfico 7: Análisis sensorial del color de los tres tratamientos de manzana deshidratada.

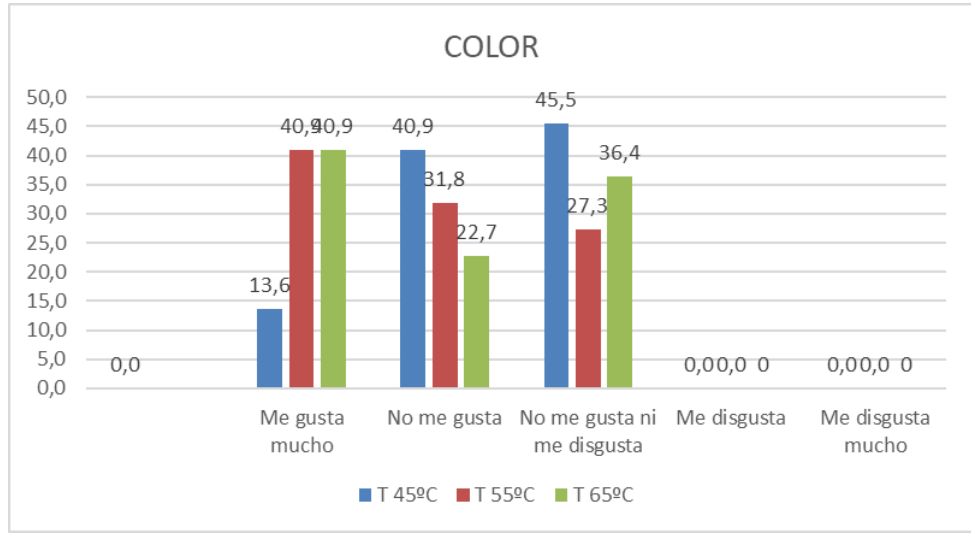




\section{Olor}

En el gráfico 8, se observa que en el tratamiento de temperatura de secado $55^{\circ} \mathrm{C}$ es el que más aceptabilidad en olor tuvo por parte del panel degustador. El 31.8\% afirmó que el olor de la fruta les gusta; el 36.4\% no les gusta ni les disgusta, y el 31.8\% les gusta mucho. Seguido del tratamiento con temperatura de secado $65^{\circ} \mathrm{C}$, el $18.2 \%$ señalo que el olor de la fruta les gusta; el $36.4 \%$ no les gusta ni les disgusta, y el 18.18\% no les gusta. (León, 2019)

Gráfico 8. Análisis sensorial del olor de los tres tratamientos de manzana deshidratada

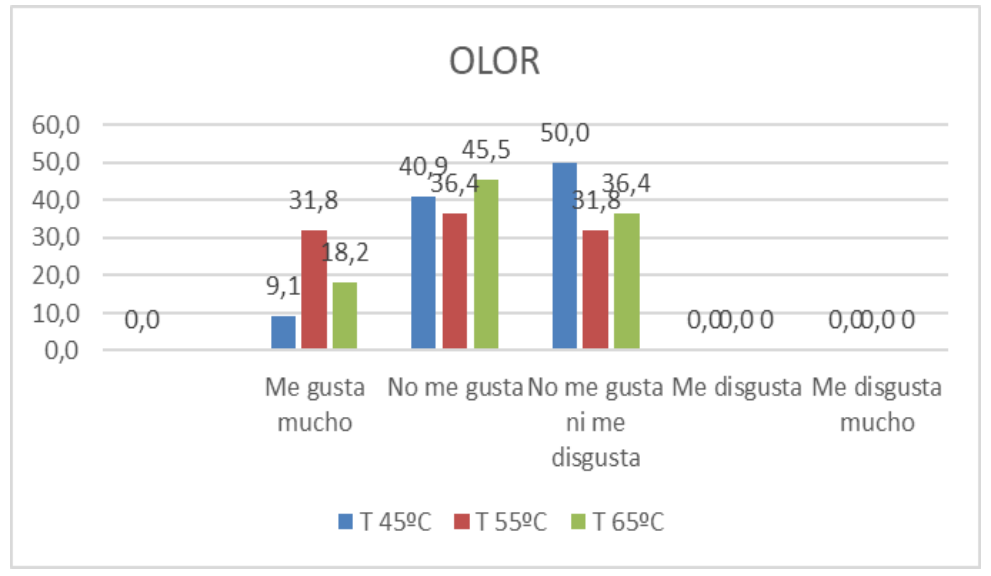

\section{Sabor}

En la gráfica 9, se observa que el tratamiento con temperatura de secado $65^{\circ} \mathrm{C}$, es el tratamiento que más aceptabilidad en sabor tuvo por parte del panel degustador; el $54.55 \%$ señalo que el sabor de la fruta deshidratada les gusta; el $27.3 \%$ indico no les gusta ni les disgusta; el 27,3\% les gusta mucho, y el 36,4\% menciona que el sabor no les gusta. (León, 2019)

Gráfico 9: Análisis sensorial del sabor de los tres tratamientos.

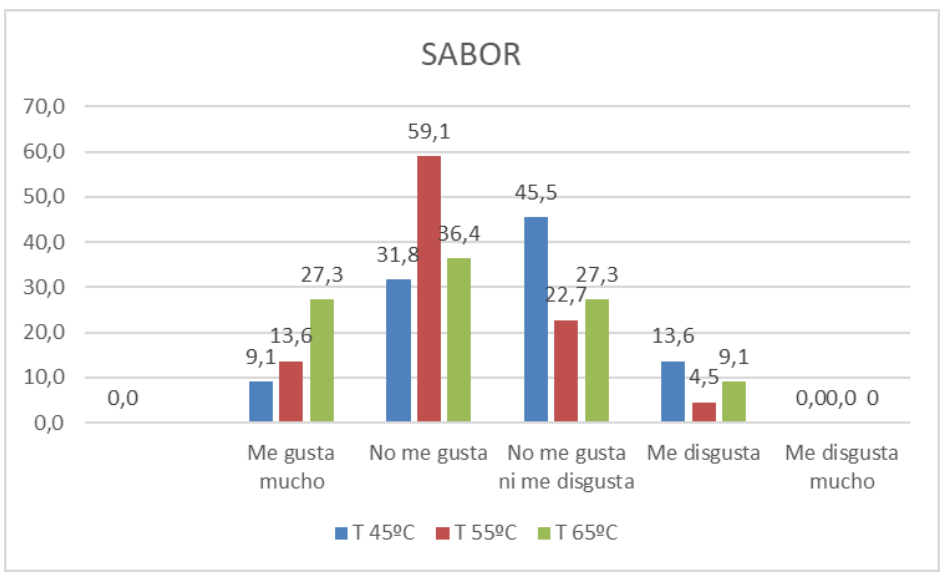




\section{Textura.}

En el gráfico 10, se observa que en el tratamiento con temperatura de secado $55^{\circ} \mathrm{C}$, es el tratamiento que más aceptabilidad en sabor tuvo por parte del panel degustador. El 22,7\% señaló que la textura de la fruta deshidratada les gusta, el 36,4\% índico no les gusta ni les disgusta, el 40,9\% les gusta mucho. (León, 2019)

Gráfico 10: Análisis sensorial de la textura de los tres tratamientos.

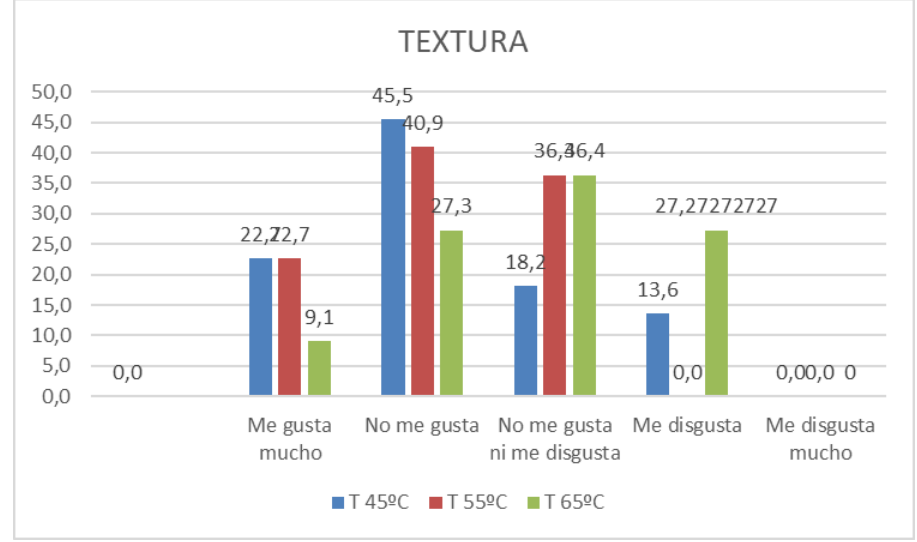

\section{Conclusiones}

- Se pudo determinar un modelo de ratio de secado utilizando el modelo matemático de la curva característica basado en la ecuación $\mathrm{y}=\mathrm{Ax}+\mathrm{B}$ en la manzana Malus Communis. $\mathrm{Sp}$; siendo la temperatura de $65^{\circ} \mathrm{C}$ la que mejor resultados presentaron en cuanto a cantidad de masa final que se obtuvo después del deshidratado.

- Las características físicas de la manzana varían en función de la humedad obteniendo un mejor sabor, color, textura y olor; variables organolépticas que se determinaron en función de la temperatura de deshidratación, siendo la temperatura de $65^{\circ} \mathrm{C}$ la de mayor aceptación.

- Se estableció la variación de la humedad en función del tiempo y la temperatura de secado en la manzana. Siendo que a una temperatura de $65^{\circ} \mathrm{C}$ se obtuvo un porcentaje de $22,4 \%$ de humedad a un tiempo de 9 horas.

- En la parte experimental del secado de manzana se determinó que el proceso debe prolongarse para conseguir mayor crocantez del producto final, a partir de las horas establecidas por el modelo los datos obtenidos no son significativos.

\section{Referencias bibliográficas.}

Alcántara, Manuel. Climafrutal.com. [En línea]. 2007 consultado: [16 de Mayo del 2019] https://climafrutal.wordpress.com/el-manzano/ 
Amador \& Domínguez. Secado Directo. [En línea]. 2009.consultado: [26 de Mayo del2019] https://1aaditiqigrupoc.wordpress.com/2009/11/06/secado-directo/

Bastidas \& Orozco. Materia Prima e industrialización.. Arte Ancestral. 1a ed. Barcelona España, 2014. pp. $30-43$

Cabezas Miguel. Evaluación nutritiva y nutraceútica de la manzana deshidratada a tres temperaturas por el método de secado de bandejas.1a ed. RiobambaEcuador,2014. pp. 130 - 143

Cabezas, Miguel. Evaluación nutritiva y nutraceútica de la manzana deshidratada a tres temperaturas por el método de secado de bandejas. 1a ed. Riobamba Ecuador, 2014. pp. $130-143$

Cerezo, Fabricio. Producción De Manzana. [En línea] 2012.consultado: [6 de Marzo del 2019] http://saludpublica.bvsp.org.bo/cc/bo40.1/documentos/704.pdf

CODEX ALIMENTARIUS. Norma general del Codex para zumos (jugos) y néctares de Frutas. Codex Alimentarius. 2005. 21 p. (Codex Stan 247)

Contreras Ángel. Características físicas de frutas deshidratadas en la Región de Huancavelica.1a ed. Lima, Perú. UNAM., 2014. pp 23 -45

Fito, P, Andrés, A., Barat, J. \& Albors, A. Introducción al secado de alimentos por aire caliente. [En línea] 2019consultado: [12 de Febrero del 2019]

Gómez, Pablo. Características de la manzana deshidratada. [En línea].2015 consultado: [22 Mayo 2019].Available at: https://www.wikiwand.com/es/caracteristicas de la manzana

Hernández Lucia. Modelo matemático del secado [En línea].2014 consultado: [10 Enero 2018]. http://apttperu.com/modelo matemático del secado/

Hernández, Lucia. Modelo matemático del secado [En línea].2014 consultado: [10 Enero 2018].httpJaramillo, A y Narváez, E. (2012). "Influencia del método de secado en parámetros de calidad relacionados con la estructura y el color de manzana y fresa deshidratadas”. Ecuador. pp 202.

Lara Luis.Píllaro de ayer y hoy.1a ed. RiobambaEcuador,2015. pp. 130 - 143

Montalván, N. \& Moyano, H. Proyecto de producción y comercialización de manzana.. 1a ed. CuencaEcuador,2009. pp. 120 - 124

Núñez Fabiola. Introducción a la deshidratación de frutas y vegetales. Segunda Edición México DF, México. Mc Graw Hill. 2014. pp 12-14

Paredes Ernesto. Estudio del proceso de horneado con microondas y su efecto sobre la estructura instrumental del fruto de cuatro variedades de manzana.[En línea] 2012consultado: [Febrero 19 2019]. Available at: http://bibdigital.epn.edu.ec/bitstream/15000/4936/1/CD-4468.pdf 
Sánchez Andrés. Manuales para educación agropecuaria cultivos de manzana área producción vegetal. 2a ed Trillas, Cali, Colombia. 2016. pp. 21://apttperu.com/modelo matemático del secado/

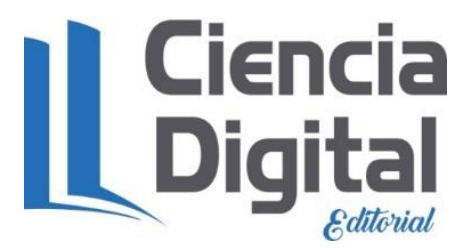


PARA CITAR EL ARTÍCULO INDEXADO.

López Sampedro, S. E., León Naranjo, A. C., Baño Ayala, D. J., \& Arboleda Alvarez, L. F. (2021). Determinación de un modelo predictivo de secado para la manzana producida en la sierra centro. ConcienciaDigital, 4(2), 247-260. https://doi.org/10.33262/concienciadigital.v4i2.1670

\section{¿Ciencia}

El artículo que se publica es de exclusiva responsabilidad de los autores y no necesariamente reflejan el pensamiento de la Revista Conciencia Digital.

El artículo queda en propiedad de la revista y, por tanto, su publicación parcial y/o total en otro medio tiene que ser autorizado por el director de la Revista Conciencia Digital.

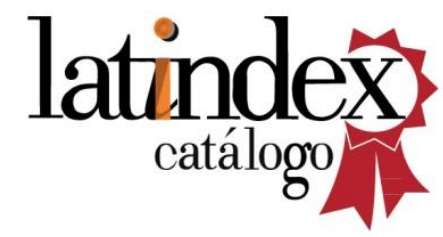

